\title{
The dynamics of subjectivity *
}

\author{
Nicholas Fleisher \\ University of Wisconsin-Milwaukee
}

\begin{abstract}
I adapt the dynamic framework for vagueness of Barker 2002 to the analysis of subjective taste predicates. I argue, following Kennedy 2013, that there are two qualitatively distinct types of subjectivity in natural language, which I call mapping subjectivity and (vague) standards subjectivity, and that the matrix predicate find is sensitive to the distinction between them. Novel to the present analysis is the proposal that find requires not just a complement that supports mapping subjectivity, but also a context that supports nonvacuous entailments about those scalar mappings. I part ways from Kennedy and from Barker 2013 in treating mapping subjectivity as a fact of the world, unassimilable to the metalinguistic variety of subjectivity associated with vague standards.
\end{abstract}

Keywords: subjectivity, vagueness, predicates of personal taste

\section{Introduction}

Recent work on the semantics of taste predicates, much of it responding to Lasersohn 2005 , has sparked interest in the nature of subjectivity and its encoding in natural language. One point of particular concern has been to understand the similarities and differences between taste predicates like tasty and vague predicates like tall: are there formal distinctions to parallel the intuitive contrast between the subjectivity involved in judge-dependent taste assessment and the subjectivity involved in the determination of vague standards, and are there linguistic diagnostics that might reveal such distinctions? Sæbø (2009) and, following him, Kennedy (2013) have seized on the behavior of the matrix predicate find as one such diagnostic, demonstrating that the felicity of sentences with find patterns consistently with the presence of taste predicates in its complement but not with the presence of ordinary vague predicates.

The matter of how best to craft a formal account of this observation is my principal concern here. The simple version of the question is "what does find need from its complement?" I follow Bouchard 2012 in rejecting Sæbø’s answer, namely that find requires its complement clause to be headed by a predicate with a bindable

* I thank the SALT attendees for helpful feedback. Special thanks to Chris Barker for extended discussion.

(C2013 Fleisher 
judge argument in the syntax. I go on to show that Bouchard's account, on which find presupposes the contingency of its subjective propositional complement, is on the right track but fails to distinguish adequately between the two types of subjectivity mentioned above. I propose instead to adapt Barker's (2002) dynamic framework for vagueness to the analysis of taste predicates, though my proposal differs in important ways from Barker's (2013) own adaptation of his 2002 framework. My answer to the question posed above is that find requires a context that supports nonvacuous judge-dependent descriptive entailments (in the sense of Barker 2002) in its complement; that is, find is used to make assertions about taste.

The broader goal, of course, is not simply to state accurately what find needs, but to understand what the behavior of find reveals about subjectivity in natural language and the proper linguistic and philosophical frameworks for analyzing it. On the former score, I will argue that the kind of subjectivity involved in taste predications is part of the world, while the kind of subjectivity involved in vague predications is metalinguistic. On the latter score, I will suggest, following Bouchard, that considerations about the syntax-semantics interface weigh in favor of a relativist treatment of taste-based subjectivity and against a contextualist treatment.

The outline of the paper is as follows. In section 2, I present the basic data on find, discuss some problems with Sæbø's contextualist analysis, and introduce some concepts and terminology for distinguishing between the two types of subjectivity. In section 3, I introduce Barker's dynamic framework and show how it allows us to capture distinctions between find and the closely related consider, while remaining consonant with the presuppositional facts discussed by Bouchard (2012). Finally, in section 4 I offer some thoughts on the grammatical and ontological status of taste judgments, arguing that they must be treated as facts about the world and not merely as objects of metalinguistic negotiation, contra Barker 2013 and Kennedy 2013.

\section{Subjectivity and gradability}

Here I provide a brief overview of the recent literature on subjectivity in taste predicates and vague predicates, with the goal of establishing the core empirical distinctions (section 2.1) and considering (and then setting aside) a prominent but unsuccessful proposal for analyzing them (section 2.2). In section 2.3, I situate these core empirical distinctions within a standard semantics of gradability, a clarifying exercise that most of the existing literature neglects to undertake.

To give the basic story arc before delving into the details: Sæbø (2009) and Kennedy (2013) proceed from the observation that natural-language subjectivity comes in at least two varieties: one associated with taste predicates, the other with ordinary vague predicates. Only the former is suitable in the complement of matrix find. Sæb $\varnothing$ takes this to indicate that the two varieties of subjective predicate are 
syntactically distinct, with those that occur felicitously in find complements containing a judge argument in the syntax. ${ }^{1}$ Bouchard (2012) provides counterexamples to most of Sæbø's arguments, casting doubt on the syntactic account of the distinction between the two types of subjectivity, though not on the underlying empirical distinction itself.

\subsection{Varieties of subjectivity}

Evidence to support the view that there are two varieties of natural-language subjectivity comes in part from "faultless disagreement" dialogues. In faultless disagreement, two speakers (or, more generally, judges) express apparently contradictory propositions (disagreement), yet neither speaker can be said to be wrong (faultlessness) (Kölbel 2002; Lasersohn 2005). ${ }^{2}$ Subjective terms that support faultless disagreement dialogues include taste predicates like tasty and ordinary vague predicates like tall, as shown in (1).

(1) Faultless disagreement:
a. A: The soup is tasty.
b. A: John is tall.
B: No, the soup is not tasty.
B: No, John is not tall.

As Kennedy (2013) observes, these two classes of adjective part ways in the comparative degree, where taste predicates continue to support faultless disagreement dialogues but ordinary vague predicates do not. The disagreement in (2a) is faultless; in $(2 b)$, by contrast, one or the other of the speakers must be wrong.

a. tastier: faultless disagreement

A: The tomato soup is tastier than the pea soup.

B: No, the tomato soup is not tastier than the pea soup.

b. taller: no faultless disagreement
A: John is taller than Bill.
B: No, John is not taller than Bill.

The disparity between the positive examples in (1) and the comparative examples in (2) reflects an intuitive distinction regarding the sources of subjectivity for the two

1 Kennedy 2012, an unpublished manuscript, endorses Sæb $\varnothing$ 's casting of the distinction in syntactic terms. Kennedy 2013 is a revised version of that manuscript with a more equivocal stance on Sæbø's syntactic proposal.

2 For an argument that such faultlessness is illusory, see Stojanovic 2007. For a formal implementation that eschews the use of judges, see Pearson 2013. For an argument that the felicity of denial is not a reliable indicator of semantic contradiction, see Sundell 2011. 
types of adjective. Whereas judges may disagree about the tastiness of a particular soup, they may not disagree about the height of an individual like John, which is an objectively verifiable fact of the world. The subjectivity found in (1b) is thus not subjectivity as regards John's height itself, but rather subjectivity as regards the operative standard of tallness. (Of course, speakers may disagree about standards of tastiness, as well.)

As Sæbø and Kennedy note, the subjective matrix verb find appears to be sensitive to this distinction, licensing only those complements that involve the kind of subjectivity found in taste predicates, as shown in (3). In this, find contrasts with its close relative consider, which licenses both kinds of subjectivity in its complement, as shown in (4).
a. I find the soup tasty.
b. \# I find John tall. ${ }^{3}$
a. I consider the soup tasty.
b. I consider John tall.

We thus have a grammatical phenomenon - the selectional sensitivity of find-to parallel the intuitive distinction between the two types of subjectivity.

\subsection{A contextualist proposal and its discontents}

Sæb $\varnothing$ uses this property of find to investigate the relative merits of relativist and contextualist analyses of taste predicates. Proceeding from Stojanovic's (2007) observation that relativist and contextualist accounts are equivalent from a purely semantic perspective, Sæbø turns to the syntax-semantics interface for evidence, ultimately endorsing a contextualist position. On Sæbø's contextualist view, taste predicates must compose syntactically with a judge argument alongside the usual individual argument (i.e., they are of type $\langle e,\langle e, t\rangle\rangle)$ and are thus typewise distinct from ordinary predicates of type $\langle e, t\rangle$. What find needs, then, is a complement of the appropriate type, with a judge argument to which the subject of find can be bound.

Sæbø advances three arguments from the syntax-semantics interface in favor of the contextualist position. I briefly sketch each of them here, along with counterexamples and refutations offered by Bouchard (2012).

3 Sæbø (2009: 328) provides the prima facie counterexample These prices may be normal for perfumes, but I still find them high. The adjective high is an ordinary vague predicate (since the amount of a given price is an objective fact), yet it can be felicitously embedded under find here. Note, though, that this use of high seems to mean not merely 'higher than the cutoff for what counts as high', but 'higher than the cutoff for what is appropriate', or 'too high for the purpose at hand'. I take these meanings to involve subjectivity that goes beyond the meaning of the adjective and the positive morpheme themselves-i.e., they involve subjectivity as regards appropriateness or purpose-and set them aside for the present discussion. (On a related positive construction, see Fleisher 2011.) 
First, Sæbø (2009: 338) claims that coordination of subjective taste predicates with nonsubjective predicates is infelicitous under find, citing examples like (5). He observes that this follows naturally on an analysis in which taste predicates and ordinary predicates are not of like type, but not on a relativist analysis in which the judge is implemented as an index of evaluation.

\# She finds him handsome and under 45.

Bouchard (2012: 191ff.), however, points out that coordination of subjective and nonsubjective predicates under find is not generally barred, citing examples like those in (6). He proposes that Sæbø's example (5) is infelicitous because it differs from the alternative She finds him handsome only in its truth-value gap, i.e., the set of worlds in which its presuppositions are not satisfied. (I refer the interested reader to Bouchard's dissertation for details.) The important point for present purposes is that the infelicity of (5) cannot be due to a type clash brought about by the syntax of coordination, since this would predict infelicity in (6) as well, contrary to fact. Indeed, Sæbø's contextualist analysis seems unable in principle to deal with examples like those in (6).

(6) a. She finds no one at the party to be handsome and under 45.

b. She finds everyone at the party to be handsome or under 45 .

Second, Sæbø (2009: 339) observes that contraposition of a nonsubjective restrictor and a subjective nuclear scope of a universal quantifier is infelicitous below find, as in (7). He suggests that this is straightforwardly derivable in a contextualist framework - find binds the judge argument of the embedded subjective main predicate unpleasant in (7a) but finds nothing to bind with nonsubjective non-smokers in (7b) — but not in a relativist one, where the judge index should be able to work its effect without regard to the internal syntax of find's complement.

(7) a. She finds all smokers to be unpleasant.

b. \# She finds all pleasant people to be non-smokers.

Bouchard (2012: 205ff.) counters that the infelicity of (7b) is due to its information structure. DPs in subject position headed by strong determiners tend to encode given information, but Bouchard suggests that there is a conflict between givenness and subjectivity, the latter requiring that the DP in question be able to have different extensions for different judges.

Third and finally, Sæbø (2009: 340) notes that the focus structure of an embedded clause can affect its acceptability under find, offering the minimal pair in (8). Sæbø suggests that the nonsubjective material in the embedded clause in (8a), but not in (8b), can be interpreted as presupposed. He sketches a DRT analysis on which 
the judge argument of beautiful remains open in the focus in (8a) and is bound by a variable introduced in the presupposition, and suggests once again that treating the judge as an index of evaluation, as on a relativist analysis, leaves one unable to account for the contrast.

(8) a. I find that you are married to a beautiful man.

b. \# I find that you know a beautiful man.

Bouchard (2012: 186ff.) disputes the notion that such focus behavior bears on the contextualism-vs.-relativism debate, claiming that find always requires that the nonsubjective material in its complement be presupposed (a matter discussed in greater detail below in section 3.3). If the embedded verb know independently resists being presupposed, as Sæbø suggests, then the infelicity of ( $8 b$ ) becomes, per Bouchard, a case of presupposition failure rather than syntactic ill-formedness.

Bouchard thus casts Sæbø's contextualist analysis of subjective taste predicatesand, in turn, of find-into serious doubt. While the basic empirical distinction between the two types of subjectivity sketched above in (1) and (2) still holds, Sæbø's contextualist account appears to miss the mark. We thus need an alternative explanation of the behavior of find. In the next section, I lay the groundwork for this by situating the two types of subjectivity within a standard semantics of gradability, a move which does much to clarify precisely where subjectivity manifests itself in taste predicates and in ordinary vague predicates.

\subsection{Judge-dependent mappings vs. vague standards}

Let us begin to make matters more precise. First, note that both taste predicates and ordinary vague predicates are gradable. Following Kennedy 1999 and much subsequent work, I take gradable predicates to denote measure functions that map an individual to a degree on a scale; composition with a degree morpheme such as positive POS or comparative -er then creates a predicate relating that individual's scalar value to some standard of comparison. In a given scalar predication, there are thus two scalar values whose determination might be subjective: (i) the value to which an individual is mapped by the measure function and (ii) the standard of comparison.

With this framework for gradability as background, we may now recast the distinction between the two types of subjectivity noted above as a distinction between subjective mappings of individuals to scalar values (e.g., how tasty a particular soup is) and subjective determination of vague standards (e.g., how tall one has to be to count as tall). Let us call the former type of subjectivity MAPPING SUBJECTIVITY and the latter type STANDARDS SUBJECTIVITY. A given sentence's subjectivity type(s) will be determined by the type of the adjective (taste predicate or ordinary 
vague predicate) in combination with the identity of the degree operator (positive or comparative). ${ }^{4}$ Faultless disagreement dialogues, in turn, are licensed wherever one or the other of the subjectivity types is present. I summarize the interactions among these factors in (9).

(9) Derivation of subjectivity type

a. The soup is tasty. (taste predicate, positive)

Mapping value $=$ the soup's tastiness (MAPPING SUBJECTIVITY)

Standard value $=$ cutoff for tasty (STANDARDS SUBJECTIVITY)

$\Rightarrow$ Subjectivity types: mapping, standards

b. John is tall. (vague predicate, positive)

Mapping value $=$ John's height (NO SUBJECTIVITY)

Standard value $=$ cutoff for tall (STANDARDS SUBJECTIVITY)

$\Rightarrow$ Subjectivity type: standards

c. The tomato soup is tastier than the pea soup. (taste predicate, comparative)

Mapping value $=$ tomato soup's tastiness (MAPPING SUBJECTIVITY)

Standard value $=$ pea soup's tastiness (MAPPING SUBJECTIVITY)

$\Rightarrow$ Subjectivity type: mapping

d. John is taller than Bill. (vague predicate, comparative)

Mapping value $=$ John's height (NO SUBJECTIVITY)

Standard value $=$ Bill's height $($ NO SUBJECTIVITY $)$

$\Rightarrow$ Subjectivity type: none

Let us reconsider the examples from (1) and (2) in light of (9). In (9a) and (9b), we see that the taste predicate tasty supports mapping subjectivity-i.e., different judges may map a given soup to different values on the scale of tastiness-but the ordinary vague predicate tall does not, as it simply maps an individual to its (judgeindependent) degree of height. Both types of predicate, however, support standards subjectivity in the positive degree; in the terminology of Kennedy \& McNally 2005, they are both relative adjectives, with the precise cutoff for what counts as tasty or tall dependent on contextual factors including subjectivity. Faultless disagreement dialogues are thus supported in both cases in (1). By contrast, in the comparatives in

4 Kennedy 2013 uses the terms EVALUATIVE and DIMENSIONAL for the classes of predicate that I call taste predicates and ordinary vague predicates, respectively. The key point is that there is a lexical semantic distinction between the two gradable adjective classes, independent of those adjectives' interactions with the various degree morphemes. 
(2), which are dissected in (9c) and (9d), the standard of comparison is provided not by the positive morpheme but by another mapping yielded by the measure function (the pea soup's tastiness or Bill's height). In (9c)/(2a), with tastier, we thus have two instances of mapping subjectivity, and so faultless disagreement is supported. In $(9 d) /(2 b)$, we have neither mapping subjectivity nor standards subjectivity, as the standard of comparison is now produced by the nonsubjective mapping of Bill to a degree of height; faultless disagreement is thus impossible here.

By incorporating basic elements of the semantics of gradability into our analysis and adopting the distinction between mapping subjectivity and standards subjectivity, we gain a better and more fine-grained way of talking about subjectivity in particular examples. We also get additional insight into the distribution of faultless disagreement dialogues in (1) and (2): such dialogues depend on the presence of at least one type of subjectivity.

Moreover, we can begin to understand the behavior of find shown in (3): it appears that find felicitously embeds only those complements that involve mapping subjectivity. Consider, by contrast, felicitously embeds both kinds of subjectivity. Confirmation of this generalization comes from examining the behavior of find and consider not just with embedded positives but also with embedded comparatives. Using the subjectivity-type derivations in (9) as a template, I show the full paradigms for find in (10) and for consider in (11). Find is felicitous only when its clausal complement supports mapping subjectivity; consider is felicitous when either mapping or standards subjectivity is present.

(10) a. I find the soup tasty.

$\Rightarrow$ Subjectivity types: mapping, standards (cf. (9a))

b. \# I find John tall.

$\Rightarrow$ Subjectivity type: standards (cf. (9b))

c. I find the tomato soup tastier than the pea soup.

$\Rightarrow$ Subjectivity type: mapping (cf. (9c))

d. \# I find John taller than Bill.

$\Rightarrow$ Subjectivity type: none (cf. (9d))

(11) a. I consider the soup tasty.

$\Rightarrow$ Subjectivity types: mapping, standards (cf. (9a))

b. I consider John tall.

$\Rightarrow$ Subjectivity type: standards (cf. (9b)) 
c. I consider the tomato soup tastier than the pea soup.

$\Rightarrow$ Subjectivity type: mapping (cf. (9c))

d. \# I consider John taller than Bill.

$\Rightarrow$ Subjectivity type: none (cf. (9d))

With our analysis of subjective terms now situated within a semantic framework for gradability, I turn to the task of fitting the analysis of subjectivity into a dynamic semantic framework. As I show in the next section, examination of a wider range of examples reveals that felicity below find depends not just on the presence of mapping subjectivity (i.e., on the lexical semantics of the adjective), but on the presence of a discourse context that supports contentful entailments about those mappings.

\section{A dynamic framework for subjectivity}

\subsection{Descriptive vs. sharpening entailments}

I begin by introducing the dynamic system of Barker 2002. Barker's analysis is designed to capture the behavior of vague predicates like tall. It does so by capitalizing on the aforementioned distinction between mapping values and standard values in scalar predication. One of Barker's core insights is that the effect of the contextual update brought about by a scalar predication will depend on whether the update narrows the context set's range of mapping values, its range of standard values, or both; i.e., the nature of the update will depend in part on the state of the input context. In adapting Barker's system to deal with taste predicates, we thus must consider what happens when the mapping values are not objective (as with tall) but subjective and tied to a judge (as with tasty). ${ }^{5}$

At the heart of Barker's (2002) analysis is the following claim: for a vague predicate in the positive degree, the value to which an individual is mapped by the measure function is a piece of information about the world, but the standard of comparison is an object of metalinguistic negotiation. An utterance of the sentence John is tall can be used to narrow the range of values of John's height in the context set (by eliminating any worlds in which John's height fails to exceed the standard in that world), or it can be used to narrow the range of values of the standard of comparison (by eliminating any worlds in which the standard meets or exceeds John's height in that world). ${ }^{6}$ The utterance can thus have what Barker calls an ordinary DESCRIPTIVE use, in which the context set is filtered according to the updated facts

5 Barker (2013) takes a somewhat different approach in adapting his 2002 system in order to deal with taste predicates. See section 4 for discussion.

6 In fact, as Barker (2002) notes, the context is typically such that an utterance of a vague predicate has both of these effects simultaneously. 
of the world, or it can have a metalinguistic SHARPENING use, in which the context set is filtered according to the updated metalinguistic usage conditions (or both).

By constraining the context set, we can block one or the other of these effects and highlight the other. Barker (2002: 2) offers the following example: if John's height is known to the interlocutors (e.g., because John is standing near them), then an utterance of John is tall...

... is not a descriptive use in the usual sense. I have not provided any new information about the world, or at least no new information about [John]'s height. In fact, assuming that tall means roughly 'having a maximal degree of height greater than a certain contextually-supplied standard', I haven't even provided you with any new information about the truth conditions of the word tall. All I have done is given you guidance concerning what the prevailing relevant standard for tallness happens to be in our community; in particular, that standard must be no greater than [John]'s maximal degree of height. ... My purpose in uttering [John is tall] under such circumstances would be nothing more than to communicate something about how to use a certain word appropriately - it would be a metalinguistic use.

If the context set is such that the mapping value of a gradable predicate as applied to a given individual is fixed - as it is with John's height in Barker's example - then an utterance of that gradable predicate in the positive degree will have no ordinary descriptive entailments. In Barker's example, the utterance of John is tall will not eliminate any live mapping values of John's height in the context set: they are all the same to begin with (modulo tolerance; see footnote 9), since John's height is an objective fact of the world and is known to the interlocutors. Instead, the utterance can have only sharpening entailments, entailments that eliminate live values of the standard of comparison. The effect of the utterance in such a context is, on this view, a purely metalinguistic one.

Barker (2002) discusses a range of linguistic phenomena that are sensitive to the distinction between descriptive and sharpening entailments. I propose to add the embedding selectivity of matrix find to the list of such phenomena. Indeed, I believe that the descriptive-vs.-sharpening distinction is the key to understanding the behavior of find. Before demonstrating this, though, we must first determine how to model the judge dependency of taste predicates in a Barker-style dynamic system. It is to this task that I now turn. 
Fleisher

\subsection{Taste mappings}

The basic question to be addressed in our dynamic analysis of taste predicates is which side of the semantic/pragmatic ledger the associated scalar mappings should be recorded on. The core empirical distinction between taste predicates and ordinary vague predicates is that, with taste predicates, the value to which a given thing is mapped by the measure function at a given world can vary from judge to judge: you might find a given soup tastier than I do. Is judge dependency of this sort a metalinguistic matter like the determination of vague standards, or are judgedependent mappings instead simply facts of the world?

I argue here that the judge-dependent mappings associated with taste predicates are facts of the world, not negotiable metalinguistic norms (contra Barker 2013; see section 4). I take no philosophical stance on what sort of facts they are (sensory percepts, opinions, something else entirely?); for linguistic purposes, all that matters is that our semantics treat them as facts of the world just as it does objective facts like John's height. The judge-dependent nature of taste predicates means, of course, that the associated scalar mappings must be indexed to a judge: there is no unique tastiness value to which a given soup is mapped at a given world, but rather a range consisting of those values to which each judge maps the soup according to his or her own taste. Once we acknowledge and control for this factor, we can show that taste mappings are qualitatively different from vague standards. ${ }^{7}$

In order to see this, let us construct a scenario similar to the one from Barker's example above. Assume a context set $c_{1}=\left\{w_{1}, w_{2}, w_{3}, w_{4}, w_{5}\right\}$, with the worlds as specified in table 1 . There are two judges $\left(j_{1}\right.$ and $\left.j_{2}\right)$, and at each world we have two values associated with each judge: the value to which the judge maps the soup on the scale of tastiness and the value that the judge takes to be the standard of tastiness (for $j_{1}$, these are $\operatorname{tast}_{j_{1}}\left(\right.$ soup) and $\mathbf{s}_{j_{1}}(\llbracket$ tasty $\rrbracket)$, respectively). Now imagine that, in context $c_{1}$, we hear the following dialogue:

$j_{1}$ : I find the soup tasty.

$j_{2}$ : I do not find the soup tasty.

This dialogue - or, more precisely, the successive contextual updates that it effectsnarrows down the context from $c_{1}$ to $c_{1^{\prime}}=\left\{w_{2}, w_{4}\right\}$, as those are the worlds in which $\operatorname{tast}_{j_{1}}($ soup $)$ exceeds $\mathbf{s}_{j_{1}}(\llbracket$ tasty $\rrbracket)$ and $\operatorname{tast}_{j_{2}}(\operatorname{soup})$ fails to exceed $\mathbf{s}_{j_{2}}(\llbracket$ tasty $\rrbracket){ }^{8}$

7 As Chris Barker (p.c.) notes, perspectival shifts in counterfactuals are unable to shift the judge in the way that they can shift indexical reference; compare If I were you, I would find the soup tasty to \#If I were you, the soup would be tasty. It is not clear to me that this is a problem for the view that judge-dependent mappings are part of the world. A good chunk of Lasersohn 2005, for instance, is devoted to detailing the differences between the grammatical behavior of judges and that of indexicals.

8 Strictly speaking, there is an intermediate context $c_{1 \text { int }}=\left\{w_{1}, w_{2}, w_{3}, w_{4}\right\}$ in between $c_{1}$ and $c_{1^{\prime}}$ that results from $j_{1}$ 's utterance. 
Dynamics of subjectivity

\begin{tabular}{c||cccc} 
& tast $_{j_{1}}$ (soup) & tast $_{j_{2}}($ soup $)$ & $\mathbf{s}_{j_{1}}(\llbracket$ tasty $\rrbracket)$ & $\mathbf{s}_{j_{2}}(\llbracket$ tasty $\rrbracket)$ \\
\hline$w_{1}$ & 85 & 60 & 50 & 50 \\
$w_{2}$ & 60 & 35 & 50 & 50 \\
$w_{3}$ & 75 & 75 & 40 & 50 \\
$w_{4}$ & 75 & 75 & 45 & 80 \\
$w_{5}$ & 40 & 30 & 55 & 45
\end{tabular}

Table $1 \quad$ tast $_{j_{1}}=$ tastiness per judge $1 ; \mathbf{s}_{j_{2}}=$ standard per judge 2; etc.

The dialogue in (12), uttered in context $c_{1}$, yields a narrowed range of both taste mappings and possible standards of tastiness. That is, on the assumption that taste mappings are facts of the world, it has both descriptive and sharpening entailments. Now imagine that, instead of $c_{1}$, we start from context $c_{2}=\left\{w_{1}, w_{2}\right\}$. In $c_{2}$, the judges agree on the standard of tastiness but differ in the tastiness values to which they map the soup. ${ }^{9}$ In $c_{2}$, the dialogue in (12) yields the updated context $c_{2^{\prime}}=$ $\left\{w_{2}\right\}$. The result is a narrowed range of taste mappings (for each judge), but no change in the range of standards of tastiness inherited from the input context: a purely descriptive usage (again, assuming that taste mappings are facts).

Let us pause to take stock of what we have so far learned about find. The dialogue in (12) has shown us that find happily embeds a taste predicate (and allows for faultless disagreement dialogues) in two kinds of contexts: (i) those that support narrowing both the range of taste mappings and the range of standards (as with $c_{1}$ ), and (ii) those that support narrowing only the range of taste mappings (as with $c_{2}$ ). What about contexts that support narrowing only the range of vague standards of tastiness, i.e., pure sharpening contexts? The context $c_{3}=\left\{w_{3}, w_{4}\right\}$ is such a context: in $c_{3}$, the two judges agree on how tasty the soup is but disagree on where the standard lies. ${ }^{10}$ To begin, note that $c_{3}$ supports pure sharpening in a canonical faultless disagreement dialogue like the one in (13).

$j_{1}$ : The soup is tasty.

$j_{2}$ : No, the soup is not tasty.

9 One might reasonably doubt whether it is indeed possible for the judges to agree, in an objectively verifiable sense, on a subjective parameter like this. I believe that what is of linguistic semantic importance for us is not that the judges agree objectively, but merely that they believe that they agree. That much, I believe, suffices to yield a context set like $c_{2}=\left\{w_{1}, w_{2}\right\}$.

Note also that, for simplicity of exposition, the toy model contexts $c_{2}$ and $c_{3}$ (below) ignore the tolerance assumption (Barker 2013: 254). I believe the account here is perfectly compatible with the tolerance assumption, though for now this remains to be demonstrated.

10 Once again, I believe that all that is necessary in order to get a context like $c_{3}$ is for the two judges to believe that they agree on the soup's tastiness, e.g., because they rated it the same on a scorecard, because they know themselves to have similar tastes in soup, or similar (cf. footnote 9). 
When uttered in $c_{3}$, the dialogue in (13) yields the updated context $c_{3^{\prime}}=\left\{w_{4}\right\}$. That is, even though the two judges agree on the tastiness value of the soup, they disagree on whether it is appropriate to call it tasty tout court: $j_{2}$ has higher standards than $j_{1}$. This is analogous to what would happen if we extended Barker's scenario above: two judges see John and one says John is tall, and then the other replies No, John is not tall. In both (13) and the extended Barker scenario, the judges are having a disagreement about the appropriate usage conditions of a gradable predicate with a vague standard in the positive degree; in both cases, the context is constrained so as only to support the sharpening entailments that are associated with this kind of metalinguistic disagreement. In this connection, note that it might be more natural in these scenarios for the second speaker to reply with I wouldn't call it tasty or I wouldn't call him tall, formulations that highlight the metalinguistic nature of the proffered denial.

Now consider what happens in context $c_{3}$ when we embed the faultless disagreement dialogue from (13) below find, i.e., when we change it to the dialogue from (12). The contrast between embedding below find and embedding below consider is particularly instructive:

a. \# find: infelicitous in $c_{3}=\left\{w_{3}, w_{4}\right\}$

$j_{1}$ : I find the soup tasty.

$j_{2}$ : I do not find the soup tasty.

b. consider: felicitous in $c_{3}=\left\{w_{3}, w_{4}\right\}$

$j_{1}$ : I consider the soup tasty.

$j_{2}$ : I do not consider the soup tasty.

The dialogues in (14) should each narrow the context to $c_{3^{\prime}}=\left\{w_{4}\right\}$, just like the unembedded faultless disagreement dialogue in (13). Evidently this is possible with consider but not with find. That is, the two judges cannot use the dialogue in (14a) in a context in which they know that they agree on the soup's tastiness value but differ in their standards; instead, they must use a dialogue like (14b), with consider, to express such a metalinguistic disagreement.

We thus see that find is not happy with a pure sharpening context like $c_{3}$. This is the case even when its complement contains a gradable predicate that supports mapping subjectivity, like tasty. The Barker-style dynamic approach lends important new insight into the nature of the subjectivity requirement associated with find: find imposes both the lexical semantic requirement that its complement support mapping subjectivity (as discussed in section 2.3) and the discourse requirement that the context support nonvacuous entailments about those mappings.

This latter requirement, in turn, supports the view that taste mappings are facts of the world and not objects of metalinguistic negotiation like vague standards. The difference between the felicity of find in $c_{2}$ and its infelicity in $c_{3}$ is illustrative: if 
taste mappings were metalinguistic, then all else being equal we would expect find to be just as infelicitous in $c_{2}$, where only entailments regarding the range of taste mappings are available, as it is in $c_{3}$. Indeed, if taste mappings were metalinguistic, then we would expect find to be equally infelicitous in $c_{1}$, where both kinds of entailment are available. If, instead, we view taste mappings as facts of the worldi.e., as facts that give rise to ordinary descriptive entailments-then the exceptional infelicity of find in the pure sharpening context $c_{3}$ immediately makes sense.

\subsection{Assertions about taste}

To summarize the preceding discussion: find requires a complement that supports mapping subjectivity in a context that supports nonvacuous entailments about those mappings. Furthermore, those entailments are not metalinguistic sharpening entailments, but ordinary descriptive entailments; i.e., subjective taste mappings are facts of the world. I take this collection of requirements to suggest an explanation that I frame for now in the form of a slogan: find is used to make assertions about taste.

We can illustrate this by considering the behavior of find in the light of Stalnaker's (1978) discussion of assertion. Stalnaker's criterion of narrowing is particularly relevant here. The narrowing criterion-i.e., the requirement that an asserted proposition have the effect of filtering some but not all worlds from the context set-must be understood in this case to do its filtering not with respect to the truth value of the entire propositional complement of find, but rather with respect to the range of mapping values associated with a given judge. The mapping values, as argued above, are facts of the world; the truth value of find's complement, by contrast, depends in part on the value of the metalinguistically negotiable positive standard of comparison (in examples like (12), that is). I take it that to make an assertion about taste is to narrow down the context set according solely to the facts of the world. This is what makes find infelicitous in context $c_{3}$ above. In $c_{3}$, the range of mapping values associated with each judge is fixed in the input context, and so there is no way to filter out some but not all of the worlds in the context set solely with respect to those mappings. Since there are no contentful descriptive entailments to be had about taste mappings in $c_{3}$, there is no contentful assertion to be made about taste. By contrast, it is perfectly possible to filter the context set in $c_{3}$ with respect to the truth value of the embedded proposition, as truth-value filtering is indifferent to the distinction between descriptive and sharpening entailments; and indeed, this is what happens with consider in (14b).

The view that find is used to make assertions about taste also serves as a useful addition to Bouchard's (2012) analysis of the presuppositional behavior of find. Bouchard observes that find presupposes the nonsubjective material in its com-

plement; for example, the sentence I find that John gave a good talk presupposes 
that John gave a talk. In order to capture this phenomenon, Bouchard (2012: 173) proposes that find triggers what he calls the Subjective Contingency Presupposition, which requires that it be possible for a judge to judge find's complement true and that it be possible for a judge to judge find's complement false. Apart from accounting for the presuppositional behavior of find, this also has the effect of deriving the mapping subjectivity requirement of find discussed above: as Bouchard shows, a complement that lacks a subjective component will violate the Subjective Contingency Presupposition due to its ineluctable failure to vary in truth value from judge to judge. ${ }^{11}$

As it stands, however, the Subjective Contingency Presupposition fails to predict the discourse requirement of find, namely that the context support nonvacuous descriptive entailments regarding the taste mappings in its complement. The Subjective Contingency Presupposition has nothing to say about why find in (12)/(14a) should be felicitous in the mixed and pure descriptive contexts $c_{1}$ and $c_{2}$ but infelicitous in the pure sharpening context $c_{3}$. By making reference only to the truth value of find's complement, Bouchard runs afoul of the truth value's aforementioned indifference to the descriptive/sharpening distinction. Indeed, the requirement that there be possible judgments making find's complement true and making it false is met in $c_{3}$-i.e., the Subjective Contingency Presupposition is satisfied-yet find is infelicitous in that context. If, by contrast, we understand find's role to be one of making assertions about taste, then we correctly predict its infelicity in $c_{3}$ : this is due to a failure of narrowing with respect to mappings, as discussed immediately above.

A fully satisfactory treatment of find might combine Bouchard's Subjective Contingency Presupposition with the present claim that find makes assertions about taste. These proposals operate at different levels - the former being a lexical semantic requirement, the latter a discourse requirement — and are compatible as far as I can tell. What I hope to have shown here is that any satisfactory treatment of find must take into account the state of the discourse, and that the Barker-style dynamic framework sketched above is a promising option.

\section{Subjectivity metalinguistic and not}

Here I address a few lingering issues regarding the relationship between the semantic framework for gradability discussed in section 2.3 and the dynamic framework discussed in section 3. I also briefly discuss the recent proposal of Barker 2013, which extends the dynamic system of Barker 2002 in a manner very different from what I have proposed above.

11 Bouchard's analysis also derives a number of correct results about presuppositions associated with quantifiers and negation embedded in the complement of find; I refer the interested reader to Bouchard's dissertation for details. 
Dynamics of subjectivity

\begin{tabular}{c||cccc} 
& tast $_{j_{1}}$ (tomato) & tast $_{j_{2}}$ (tomato) & tast $_{j_{1}}($ pea $)$ & tast $_{j_{2}}($ pea $)$ \\
\hline$w_{6}$ & 75 & 75 & 40 & 50 \\
$w_{7}$ & 75 & 75 & 45 & 80
\end{tabular}

Table $2 \operatorname{tast}_{j_{1}}($ tomato $)=$ tastiness of tomato soup per judge 1; etc.

In section 2.3 I sketched a distinction between mapping subjectivity and standards subjectivity. I went on in section 3 to argue that the judge-dependent scalar mappings involved in mapping subjectivity are associated with ordinary descriptive entailments, while the vague standards involved in standards subjectivity are associated with metalinguistic sharpening entailments. It is important to keep in mind that not all standards of comparison are vague standards like those found with relative adjectives (Kennedy \& McNally 2005) in the positive degree. Indeed, in (9c) we saw that the standard of comparison with a taste predicate in the comparative degree supports mapping subjectivity, not standards subjectivity (which I henceforth call vaguestandards subjectivity, in the interest of terminological clarity). Translated into our dynamic framework, this means that not all standards of comparison are associated with metalinguistic sharpening entailments: only the vague standards associated with relative adjectives in the positive degree give rise to sharpening.

If we examine taste predicates in the comparative degree, we discover that find can in fact be used in contexts where the judges agree on the mapping value of the subject of taste predication. For example, in context $c_{4}=\left\{w_{6}, w_{7}\right\}$, with the worlds specified as in table 2 , the dialogue in (15) is perfectly felicitous.

$j_{1}$ : I find the tomato soup tastier than the pea soup.

$j_{2}$ : I do not find the tomato soup tastier than the pea soup.

The dialogue in (15) successfully narrows the input context $c_{4}$ to the updated context $c_{4^{\prime}}=\left\{w_{7}\right\}$. This is despite the fact that the various scalar values involved in $c_{4}$ are equivalent to those in $c_{3}$, where find was infelicitous. The crucial difference, of course, is that the vague standards of tastiness $\mathbf{s}_{j_{1}}(\llbracket$ tasty $\rrbracket)$ and $\mathbf{s}_{j_{2}}(\llbracket$ tasty $\rrbracket)$ from $c_{3}$ have been replaced with the mapping values $\operatorname{tast}_{j_{1}}(p e a)$ and $\operatorname{tast}_{j_{2}}($ pea $)$ in $c_{4}$. Whereas positive scalar predications with taste predicates (as in (12)) involve comparing a judge-dependent mapping value (a fact of the world) to a vague standard (an object of metalinguistic negotiation), comparative scalar predications with taste predicates (as in (15)) involve comparing two judge-dependent mapping values (two facts of the world). In the case at hand, these are the degree to which the judge finds the tomato soup tasty and the degree to which the judge finds the pea soup tasty.

The reason for the felicity of the dialogue in (15) should be perfectly clear. The contextual update from $c_{4}$ to $c_{4^{\prime}}$-i.e., the narrowing from $\left\{w_{6}, w_{7}\right\}$ to $\left\{w_{7}\right\}$-is 
an instance of narrowing with respect to the standards of comparison $\operatorname{tast}_{j_{1}}(p e a)$ and $\operatorname{tast}_{j_{2}}$ (pea). Unlike dialogue (12) in $c_{3}$, dialogue (15) in $c_{4}$ involves standards of comparison that are judge-dependent mappings of an object to a value on the scale; i.e., the standards of comparison are facts of the world, not metalinguistically negotiable norms. It is thus perfectly felicitous to use dialogues like (15), with find, in contexts like $c_{4}$, despite the fact that the judges agree on the mapping value for the embedded subject. As long as the judges disagree on the mapping value for the standard of comparison, judge-dependent descriptive entailments are supported, and we can use find to make assertions about taste.

These considerations reinforce the idea that there is a qualitative difference between the subjectivity involved in judge-dependent scalar mappings and the subjectivity involved in the determination of vague standards associated with the positive degree (this is also the conclusion of Kennedy 2013, though he does not explore the dynamic/discourse factors examined here). As the data from comparatives show, find requires a context that supports nonvacuous entailments about mapping values, regardless of whether those entailments involve narrowing with respect to the embedded subject's mapping value or with respect to the standard of comparison. Put another way, the crucial factor that renders a context inhospitable for find is not a limitation to narrowing with respect to the standard of comparison, but a limitation to narrowing with respect to a vague, positive standard of comparison. As I have argued above, we can make sense of this by treating the offending entailments about vague standards as metalinguistic sharpening entailments, while treating entailments about judge-dependent scalar mappings (whether standards or not) as ordinary descriptive entailments. If find is used to make assertions about taste, then it requires a context that supports the latter kind of entailment.

The bifurcated perspective on subjectivity developed here is drastically different from the one advanced by Barker (2013), who aims to bring all subjectivity into the metalinguistic fold. Barker (2013: 250) writes of taste predicates that they involve "norms for which certain aspects of an object are relevant for the judgment in question, ... as well as the norms for convolving the relevant aspects into a judgment of the degree to which the object possesses the property in question." He goes on to suggest that "such norms constitute part of the discourse, rather than being part of the world under discussion." Kennedy (2013: §4), though he recognizes find's sensitivity to the distinction between what I have called mapping subjectivity and vague-standards subjectivity, pursues a similar unifying line of explanation:

[J]ust as there is uncertainty about how the dimensions involved in vague standard calculation are weighted and applied in different conversational situations, there is uncertainty about how the dimensions of qualitative assessment are weighted and applied by different 
Dynamics of subjectivity

judges. In the former case, the uncertainty leads to uncertainty about standards; in the latter case, it leads to uncertainty about orderings.

I believe that these attempts to assimilate mapping subjectivity to vague-standards subjectivity fly in the face of intuition. While it is certainly possible for judges to disagree about the norms or dimensions of assessment governing the appropriate use of a taste predicate, I believe that judge-dependent variation in the experience of taste is an irreducible fact of the world, one directly reflected in the mapping of objects to values on the associated scale by a given judge. A dispute about which of two soups is tastier need not be a dispute rooted in metalinguistic uncertainty about the normative usage of the word tasty; it can simply be a dispute about how the world is. By contrast, a dispute about whether John (whose height is common knowledge) is tall is inevitably a metalinguistic dispute about the normative usage of the positive form tall. As we have seen, the distinction is supported by an independent linguistic diagnostic: the sensitivity of the matrix verb find.

One of Barker's (2013) goals in proposing that all subjectivity is metalinguistic is to explore the possibility of accounting for subjectivity without resort to a relativist treatment like that of Lasersohn 2005. I have little to add to what others have said on this score, but I believe that Bouchard (2012), in weighing considerations from the syntax-semantics interface, makes a strong case against the contextualist account of Sæbø 2009 and in favor of relativism (see section 2.2). The discourse conditions on the felicity of find that we have examined are likewise consistent with a relativist treatment: it is clear from our discussion that a predicate's supporting mapping subjectivity is a necessary but not a sufficient condition for embedding below find (think of (12) in $c_{1}$ vs. $c_{3}$ ). It thus strikes me as unlikely that the contextualist's syntactic/type-theoretic framing of what find needs can offer a satisfactory account.

Certainly there remain many open questions regarding subjectivity, the requirements of find, and the account explored here. One issue that space considerations preclude me from examining concerns the specifics of narrowing in assertions about taste. Must taste assertions narrow the range of live mapping values associated with a particular judge, or is it sufficient for such an assertion to filter out worlds with mapping values in the middle of the input range (i.e., because said mapping value fails to exceed the standard in that world)? Are there standards associated with each judge, as I have sketched in tables 1 and 2, or is there a single standard in each world, as Barker (2013) suggests? Detailed investigation of these issues must await future research. For now, I hope simply to have shown that the qualitative distinction between judge-dependent mapping subjectivity and vague-standards subjectivity is thoroughgoing and real, and that a dynamic treatment offers some insight into find's sensitivity to that distinction. 


\section{References}

Barker, Chris. 2002. The dynamics of vagueness. Linguistics and Philosophy 25. $1-36$.

Barker, Chris. 2013. Negotiating taste. Inquiry 56. 240-257.

Bouchard, David-Étienne. 2012. Long-distance degree quantification and the grammar of subjectivity. Montréal: McGill University Ph.D. dissertation.

Fleisher, Nicholas. 2011. Attributive adjectives, infinitival relatives, and the semantics of inappropriateness. Journal of Linguistics 47. 341-380.

Kennedy, Christopher. 1999. Projecting the Adjective: The Syntax and Semantics of Gradability and Comparison. New York: Garland.

Kennedy, Christopher. 2012. Two kinds of subjectivity. Ms., University of Chicago.

Kennedy, Christopher. 2013. Two sources of subjectivity: Qualitative assessment and dimensional uncertainty. Inquiry 56. 258-277.

Kennedy, Christopher \& Louise McNally. 2005. Scale structure, degree modification, and the semantics of gradable predicates. Language 81. 345-381.

Kölbel, Max. 2002. Truth Without Objectivity. London: Routledge.

Lasersohn, Peter. 2005. Context dependence, disagreement, and predicates of personal taste. Linguistics and Philosophy 28. 643-686.

Pearson, Hazel. 2013. A judge-free semantics for predicates of personal taste. Journal of Semantics 30. 103-154.

Sæbø, Kjell Johan. 2009. Judgment ascriptions. Linguistics and Philosophy 32. 327-352.

Stalnaker, Robert C. 1978. Assertion. In Peter Cole (ed.), Syntax and Semantics: Pragmatics, vol. 9, 315-322. San Diego: Academic Press.

Stojanovic, Isidora. 2007. Talking about taste: Disagreement, implicit arguments, and relative truth. Linguistics and Philosophy 30. 691-706.

Sundell, Timothy. 2011. Disagreements about taste. Philosophical Studies 155. 267-288.

Nicholas Fleisher

Department of Linguistics

University of Wisconsin-Milwaukee

P.O. Box 413

Milwaukee, WI 53201-0413

fleishen@uwm.edu 